\title{
Metformin attenuates angiotensin II-induced cardiomyocyte hypertrophy by upregulating the MuRF1 and MAFbx pathway
}

\author{
FAWANG DU $^{1}$, YALIN CAO ${ }^{1}$, YAN RAN $^{2}$, QIANG WU $^{1}$ and BAOLIN CHEN ${ }^{1}$ \\ Departments of ${ }^{1}$ Cardiology and ${ }^{2}$ Nephrology, Guizhou Provincial People's Hospital, Guiyang, Guizhou 550002, P.R. China
}

Received July 28, 2020; Accepted December 8, 2020

DOI: $10.3892 / \mathrm{etm} .2021 .10665$

\begin{abstract}
Pathological cardiac hypertrophy induced by aging and neurohumoral activation, such as angiotensin II (Ang II) activation, is an independent risk factor for heart failure. The muscle really interesting new gene-finger protein-1 (MuRF1) and muscle atrophy F-box (MAFbx) pathway has been previously reported to be an important mechanism underlying the pathogenesis of cardiac hypertrophy. Metformin is currently the first-line blood glucose-lowering agent that can be useful for the treatment of cardiovascular diseases. However, the potential role of metformin in the modulation of MuRF1 and MAFbx in cardiomyocyte hypertrophy remains poorly understood. The present study used H9c2 cells, a cardiomyocyte cell model. The surface area of cultured rat $\mathrm{H} 9 \mathrm{c} 2$ myoblasts was measured and the expression levels of MuRF1 and MAFbx were quantified using western blot or reverse transcription-quantitative PCR. H9c2 cells were transfected with MuRF1 and MAFbx small interfering (si) RNA. The present study revealed that Ang II treatment significantly increased the cell surface area of model cardiomyocytes. Additionally, atrial natriuretic peptide (ANP) and brain natriuretic peptide (BNP) mRNA and protein expression was increased following this treatment. Ang II also downregulated MuRF1 and MAFbx protein and mRNA expression. In the $\mathrm{H} 9 \mathrm{C} 2$, treatment with metformin attenuated hypertrophic remodeling. In addition, expression of ANP and BNP was significantly reduced in metformin-treated H9C2 cells. The results indicated that metformin increased the activity of MuRF1 and MAFbx and upregulated their expression, the knockdown of which resulted in deteriorative Ang II-induced cell hypertrophy, even following treatment with metformin. Taken together, data from the present study suggest that metformin can prevent cardiac hypertrophy through the MuRF1 and MAFbx pathways.
\end{abstract}

Correspondence to: Dr Baolin Chen, Department of Cardiology, Guizhou Provincial People's Hospital, 83 Zhongshan Dong Road, Guiyang, Guizhou 550002, P.R. China

E-mail: chenbl104@126.com

Key words: metformin, cardiomyocyte hypertrophy, muscle really interesting new gene-finger protein-1, muscle atrophy F-box

\section{Introduction}

Cardiac hypertrophy is an adaptive response to stress and occurs when the individual cellular volume of the heart increases, an effect that is associated with neurohumoral reactivation (1). In almost all types of cardiac disease, cardiac hypertrophy is a critical adaptive response in cardiomyocytes that aims to preserve the output and efficiency of the heart in addition to optimize cardiac pump function (2). However, sustained cardiac hypertrophy is associated with myocardial remodeling and dilatation of the left ventricle, which can ultimately lead to dilated cardiomyopathy, arrhythmia, fibrotic disease and heart failure (3). At cellular level, cardiac hypertrophy is characterized by enlarged cell size and increased synthesis of various cardiac hypertrophy markers, including atrial natriuretic peptide (ANP) and brain natriuretic peptide (BNP) (4).

The ubiquitin-proteasome system (UPS) protects cardiomyocytes by preventing the build-up of misfolded proteins and by removing pro-apoptotic signaling molecules (5). It has been previously demonstrated that protein degradation via the UPS is activated in animal models of cardiac hypertrophy and in cases of human cardiomyopathy) (6). In addition, UPS dysfunction can result in cardiac hypertrophy and defective cardiac responses (7). Muscle really interesting new gene (RING)-finger protein-1 (MuRF1) and muscle atrophy F-box (MAFbx) are atrogenes that serve as early markers of skeletal muscle atrophy and aid in the diagnosis of muscle disease (8). Previous studies have determined an association between Atrogin-1/MAFbx with cardiac hypertrophy, such that MuRF1 and MAFbx have been reported to be negative regulators of cardiac hypertrophy $(9,10)$. However, the precise mechanism underlying the involvement of MuRF1 and MAFbx in cardiac hypertrophy pathogenesis remains to be fully elucidated.

Metformin is currently the first-line blood glucose-lowering agent for the treatment of type 2 diabetes mellitus, with potential for future application in cardiovascular diseases (11). It inhibits hepatic gluconeogenesis, enhances glucose uptake in peripheral tissues and reduces glucose absorption from the intestine (12). Furthermore, experimental studies have previously revealed that metformin treatment can effectively reverse infarct size, reduce vascular inflammation and myocardial injury after ischemia, inhibit cardiomyocyte apoptosis and protect against ischemia/reperfusion-induced cardiomyocyte death $(13,14)$. 
Therefore, considering the potential role of metformin in the modulation of MuRF1 and MAFbx during angiotensin II (Ang II)-induced cardiomyocyte hypertrophy, the present study aimed to determine the effects of metformin on cardiomyocyte hypertrophy, with the research focus on the MuRF1 and MAFbx pathway, in an in vitro model of Ang II-induced cardiomyocyte hypertrophy.

\section{Materials and methods}

Cell culture and treatment. Rat $\mathrm{H} 9 \mathrm{c} 2$ myoblasts, a model for cardiomyocytes, were purchased from the Cell Bank of Type Culture Collection of the Chinese Academy of Sciences. These cells were routinely authenticated and tested negative for Mycoplasma contamination and were cultured in DMEM (Gibco; Thermo Fisher Scientific, Inc.) supplemented with $1 \%$ streptomycin-penicillin and 10\% FBS (Gibco; Thermo Fisher Scientific, Inc.) and $4 \mathrm{mM}$ L-glutamine (Gibco; Thermo Fisher Scientific, Inc.) in in an atmosphere of $5 \% \mathrm{CO}_{2}$ at $37^{\circ} \mathrm{C}$. Cells were collected and centrifuged at $168 \mathrm{x} \mathrm{g}$ for 5 min at $18^{\circ} \mathrm{C}$, after which the supernatant was discarded. The complete medium was changed every 3 days and cells were then sub-cultured when the density reached $80 \%$ confluence. After incubation, cells were washed three times with PBS and harvested by digestion with trypsin. The cells were then placed into a single cell suspension, after which $1 \times 10^{5}$ cells $/ \mathrm{ml}$ were seeded into six-well plates. The $\mathrm{H} 9 \mathrm{c} 2$ cardiomyocytes in all the experiments were passaged according to the ratio of 1:3 at $37^{\circ} \mathrm{C}$ and $5 \% \mathrm{CO}_{2}$ in saturated humidity conditions to expand cultivation. Cells were subsequently divided into different groups according to the experimental requirements.

To evaluate the effects of metformin on cardiomyocyte hypertrophy, cultured cells were randomly divided into the following four experimental groups: i) Control, untreated $\mathrm{H} 9 \mathrm{c} 2$ cells incubated in $10 \% \mathrm{FBS}$ DMEM for $24 \mathrm{~h}$ at $37^{\circ} \mathrm{C}$; ii) metformin, where cells were treated with $2 \mathrm{mM}$ metformin (Sigma-Aldrich; Merck KGaA) for $24 \mathrm{~h}$ at $37^{\circ} \mathrm{C}(15)$; iii) Ang II, where cells were treated with $100 \mathrm{nM}$ Ang II (Beijing Solarbio Science \& Technology Co., Ltd.) for $48 \mathrm{~h}$ at $37^{\circ} \mathrm{C}$; and iv) Ang II + metformin, where cells were treated with $100 \mathrm{nM}$ Ang II for $48 \mathrm{~h}$, followed by metformin for $24 \mathrm{~h}$ at $37^{\circ} \mathrm{C}$.

To determine the role of MuRF1 and MAFbx after metformin treatment in this angiotensin II-induced cardiomyocyte hypertrophy model, cells were randomly divided into the following seven experimental groups: i) Control; ii) metformin, treated with $2 \mathrm{mM}$ metformin for $24 \mathrm{~h}$ at $37^{\circ} \mathrm{C}$; iii) Ang II, treated with $100 \mathrm{nM} \mathrm{Ang} \mathrm{II} \mathrm{for} 48 \mathrm{~h}$ at $37^{\circ} \mathrm{C}$; iv) Ang II + metformin, treated with $100 \mathrm{nM} \mathrm{Ang} \mathrm{II} \mathrm{for} 48 \mathrm{~h}$, followed by metformin for $24 \mathrm{~h}$ at $37^{\circ} \mathrm{C}$; v) Ang II + Met + NC-siRNA groups; vi) Ang II + metformin + MAFbx-siRNA; and vii) Ang II + metformin + MuRF1-siRNA. Groups v, vi and vii were first treated with $100 \mathrm{nM}$ Ang II for $48 \mathrm{~h}$, followed by metformin for $24 \mathrm{~h}$. After siRNA targeting MuRF1 and MAFbx was used to knock down MuRF1 and MAFbx expression, changes in the surface area and the protein degradation rate in cells were examined.

Cell surface area measurement. To determine changes in cell size, cells $(n=100)$ were randomly selected from each experimental group. Images of 3 fields per dish were captured with an average of 30-40 cells per field. and imaged using an Olympus BX51 microscope (Olympus Corporation) at a magnification of $x 200$. The cell surface area was measured using the Image-J, 1.37v Image software (National Institutes of Health).

Reverse transcription-quantitative PCR (RT-qPCR). Total RNA was extracted using TRIzol ${ }^{\circledR}$ reagent according to manufacturer's protocol (Thermo Fisher Scientific, Inc.). The RNA sample $(5 \mu \mathrm{g})$ was treated with DNase and subsequently used for cDNA synthesis. The mRNA was reverse transcribed into cDNA using HiScript ${ }^{\circledR}$ II 1st Strand cDNA Synthesis Kit (Vazyme Biotech Co., Ltd; cat. no. R101-01/02) according to the manufacturer's protocol. qPCR was performed in Applied Biosystems QuantStudio 6 Flex Real-Time PCR System (Thermo Fisher Scientific, Inc.) using SYBR ${ }^{\circledR}$ Green Master Mix (Vazyme Biotech Co., Ltd.; cat. no. Q111-02) under the following thermocycling conditions: Initial denaturation at $95^{\circ} \mathrm{C}$ for $5 \mathrm{~min}$, followed by 40 cycles at $94^{\circ} \mathrm{C}$ for $30 \mathrm{sec}, 60^{\circ} \mathrm{C}$ for $30 \mathrm{sec}$ and $72^{\circ} \mathrm{C}$ for $1 \mathrm{~min}$. To assess the concentration and purity of total RNA, the optical density (OD) 260: OD 280 (1.8:2.0) ratio of the extracted RNA sample was measured. The concentration of RNA was subsequently calculated according to the OD value, with use of the following formula: Total RNA concentration $(\mu \mathrm{g} / \mu \mathrm{l})=\mathrm{OD} 260 \times 40 \times 10^{-3}$. The following primers were used: Rat $\beta$-actin forward, 5'-CACGATGGAGG GGCCGGACTCATC-3' and reverse, 5'-TAAAGACCTCT ATGCCAACACAGT-3'; rat MAFbx forward, 5'-TCCCTGAG TGGCATCGCCCAAAAGA-3' and reverse, 5'-ACACAGGCA GGT CGGTGATCGT-3'; rat MuRF1 forward, 5'-GGTGCCTA CTTGCTCCTTGTGC-3' and reverse, 5'-AGTCTGAACTCGGTCG TTCCCT-3'; rat ANP forward, 5'-GGAAGTCAACCCG TC TCA-3' and reverse, 5'-GGGCTCCAATCCTGTCAAT-3' and rat BNP forward, 5'-AGATGATTCTGCTCCTGCTTT-3' and reverse, 5'-TGAGCCATTTCCTCTGACTTT-3'. The PCR reactions yielded 240, 286, 145, 167 and 145 bp fragments for $\beta$-actin, MAFbx, MuRF1, MuRF1, ANP and BNP, respectively. The relative gene expression was normalized to that of the house keeping gene $\beta$-actin using the $2^{-\Delta \Delta \mathrm{Cq}}$ method (16).

Western blotting. H9C2 cells were lysed in RIPA buffer (Shanghai Biyuntian Biotechnology Co., Ltd.) containing a mixture of protease inhibitors (Puregene AG) and the total protein content of each sample and maker was determined using the bicinchoninic acid protein assay kit (Puregene AG). Each protein sample was diluted 20 times, after which standard protein dilutions were prepared using bovine serum albumin $(2 \mathrm{mg} / \mathrm{ml})$ in duplicate at concentrations of 1, 0.8, 0.6, 0.4 and $0.2 \mathrm{mg} / \mathrm{ml}$. An equal amount of protein was added per lane $(40 \mu \mathrm{g})$, separated by $10 \%$ SDS-PAGE and transferred onto PVDF membranes.

The membranes were blocked with $5 \%$ skimmed milk powder in Tris buffered saline with $0.5 \%$ Tween-20 (TBS-T) at room temperature for $2 \mathrm{~h}$. The primary antibodies used for western blotting were as follows: ANP (1:1,000; cat. no. DF6497; Affinity Biosciences, Ltd.), MuRF1 (E3 Ub-ligases TRIM63; 1:1,000; cat. no. DF7178; Affinity Biosciences, Ltd.), MAFbx (1:800; cat. no. DF7075; Affinity Biosciences, Ltd.), $\beta$-actin (1:500; cat. no. BM0627; Wuhan Boster Biological 

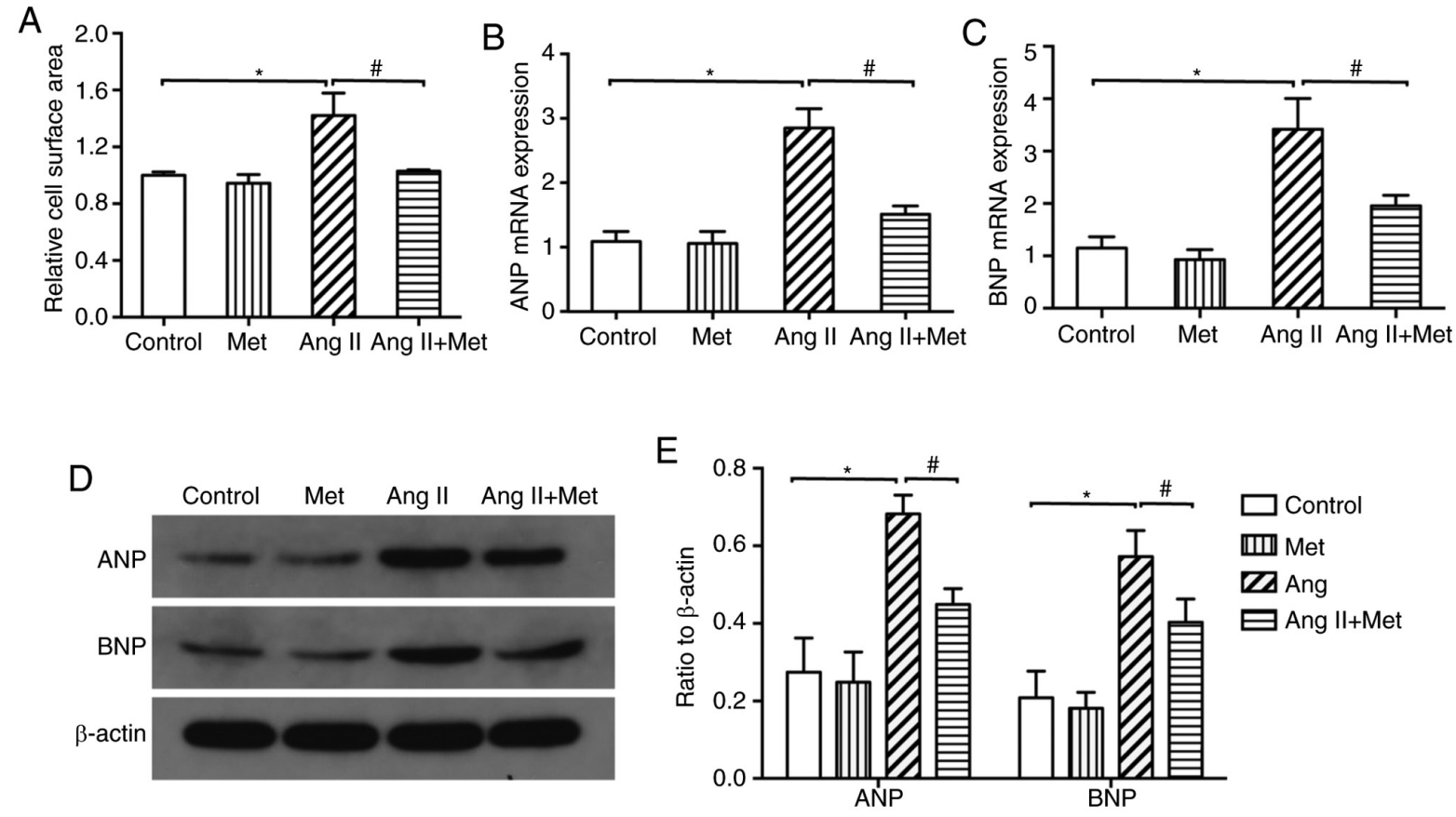

Figure 1. Effect of metformin on cardiomyocyte hypertrophy. (A) Metformin reversed the changes in the surface area of cardiomyocytes induced by Ang II treatment for $48 \mathrm{~h}$. Metformin reversed the changes in (B) ANP and (C) BNP mRNA expression in cardiomyocytes after treatment with Ang II for $48 \mathrm{~h}$. (D) Western blot analysis of ANP and BNP. (E) Densitometric analysis of ANP and BNP expression in cardiomyocytes. $\mathrm{n}=3$ for each group. ${ }^{*} \mathrm{P}<0.05 .{ }^{~} \mathrm{P}<0.05$. ANP, atrial natriuretic peptide; BNP, brain natriuretic peptide; Ang, angiotensin; Met, metformin.

Technology, Ltd.) and BNP (1:500; cat. no. ab19645; Abcam). Primary antibody incubation was performed at $4^{\circ} \mathrm{C}$ overnight. After washing in TBS-T five times, the membrane was incubated with horseradish-peroxidase-conjugated secondary antibodies (1:50,000; Wuhan Boster Biological Technology, Ltd.; cat. no. BA1051 and BA1051) at room temperature for 2 h. Protein bands were detected by an enhanced-chemiluminescent reagent (Vazyme Biotech Co., Ltd.) and exposed to $\mathrm{X}$-ray film. Band density was analyzed and quantified using the Image-J imaging software $(1.37 \mathrm{v}$; National Institutes of Health).

MuRF1 and MAFbx knockdown. The MuRF1 and MAFbx small interfering (si) RNA oligonucleotides (20 nM) were purchased from Thermo Fisher Scientific, Inc. H9c2 cells were first seeded at a density of $5 \times 10^{5}$ cells/well into 6 -well plates and were transfected using Opti-MEM (Gibco; Thermo Fisher Scientific, Inc.) containing Lipofectamine ${ }^{\circledR}$ RNAiMAX (Invitrogen; Thermo Fisher Scientific, Inc.). Cells were updated with fresh DMEM medium supplemented with 2\% FBS after transfection $4 \mathrm{~h}$ before being subjected to either treatments or assays for knockdown efficiency, and then collected $24 \mathrm{~h}$ later. The following siRNA sequences were used: MAFbx-851 siRNA sense, 5'-GGCAGCUGGAUUGGAAGAATT-3' and antisense, 5'-UUCUUCCAAUCCAGCUGCCTT-3'; MuRF1-327 siRNA sense, 5'-GCCGCCAUGAAGUGAUCAUTT-3' and antisense, 5'-AUGAUCACUUCAUGGCGGCTT-3'; negative control (NC)-siRNA sense, 5'-UUCUCCGAACGUGUCACGUTT -3' and antisense, 5'-ACGUGACACGUUCGGAGAATT-3'.

Statistical analysis. A total of three independent experimental repeats were carried out for each experiment and data are presented as the mean \pm SEM and analyzed using one-way ANOVA followed by Bonferroni post hoc test with the SPSS 22.0 statistical software (IBM Corp.). $\mathrm{P}<0.05$ was considered to indicate a statistically significant difference.

\section{Results}

Inhibitory effects of metformin on cardiomyocyte hypertrophy. Treatment with Ang II was found to significantly increase the surface area of cardiomyocytes compared with those in control (Figs. 1 and 2). Additionally, compared with those in control, protein and mRNA expression levels of ANP and BNP were significantly increased by Ang II treatment $(\mathrm{P}<0.05$; Fig. 1B and $\mathrm{C})$. Subsequent experiments demonstrated that the Ang II-induced increments in cardiomyocyte surface area, mRNA and protein expression levels of ANP and BNP were all significantly reversed by metformin treatment for $24 \mathrm{~h}(\mathrm{P}<0.05$; Fig. 1). These results suggest that metformin can attenuate Ang II-induced cardiomyocyte hypertrophy.

Effects of metformin on MuRF1 and MAFbx expression. To investigate if MuRF1 and MAFbx are involved in the attenuation of Ang II-induced cardiomyocyte hypertrophy by metformin, the effects of Ang II and metformin on the protein expression of MuRF1 and MAFbx were assessed. The results revealed that compared with those in the control group, Ang II treatment significantly downregulated MuRF1 and MAFbx protein expression levels in cultured cardiomyocytes $(\mathrm{P}<0.05)$. However, metformin treatment significantly reversed this reduction in MuRF1 and MAFbx protein expression in Ang II stimulated cells $(\mathrm{P}<0.05$; Fig. 3).

MuRF1-siRNA and MAFbx-siRNA blocks the antihypertrophic effects of metformin. Transfection with MuRF1-siRNA 
Control

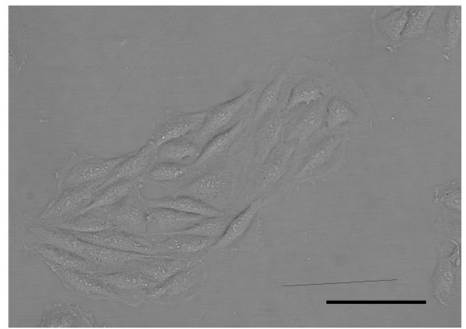

Ang II+Met

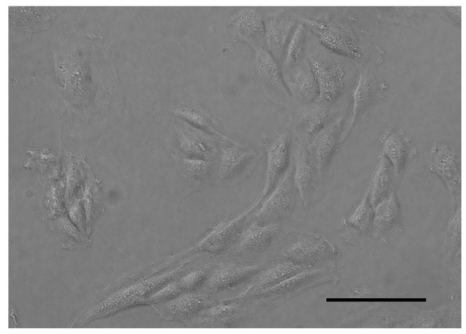

Ang II +Met + siRNA-MAFbx
Met

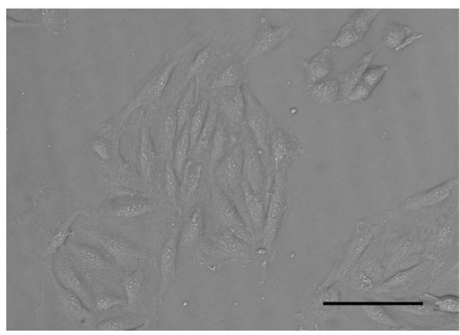

Ang II+Met+NC-siRNA

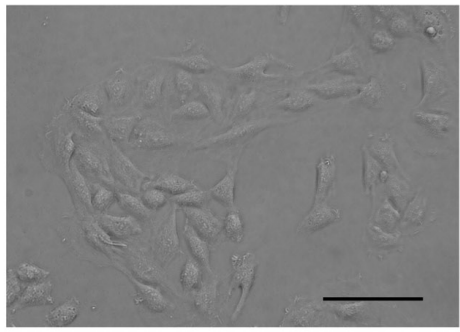

Ang II

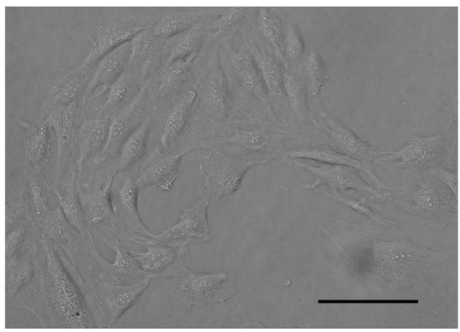

Ang II +Met + siRNA-MuRF1

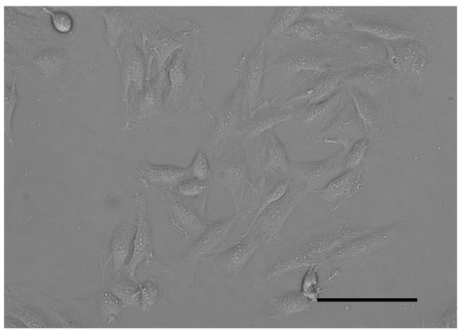

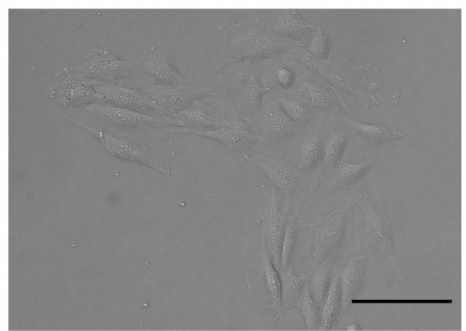

Figure 2. Representative images illustrating changes in cardiomyocyte surface area. Metformin reduced the surface area of cardiomyocytes. MuRF1-siRNA and MAFbx-siRNA increases cardiomyocyte size in the presence of metformin. Scale bar, $100 \mu \mathrm{m}$. MuRF1, muscle really interesting new gene-finger protein-1; MAFbx, muscle atrophy F-box; siRNA, small interfering.
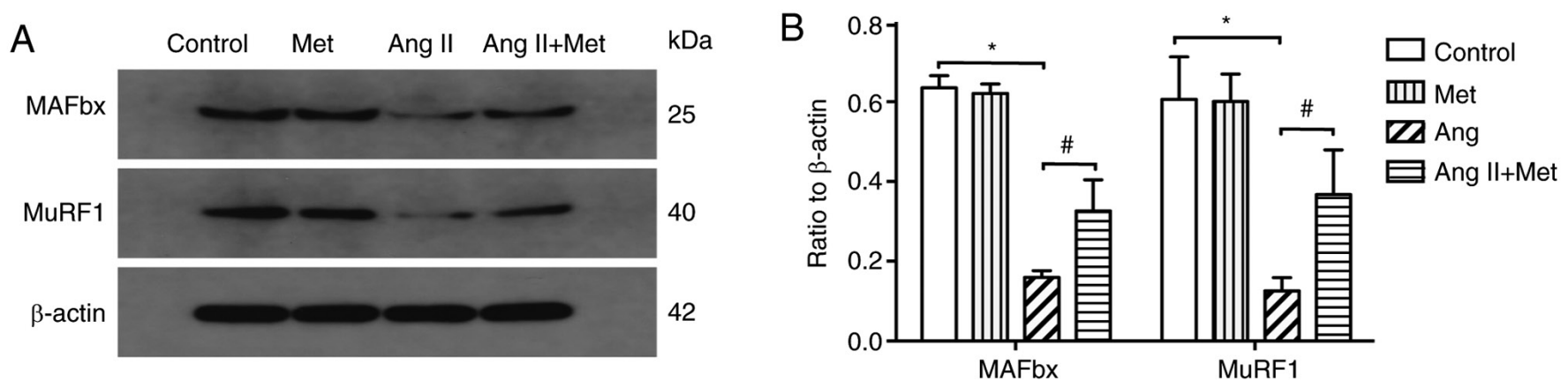

Figure 3. Effects of metformin on MuRF1 and MAFbx expression. (A) Western blot analysis measuring the expression of MuRF1 and MAFbx protein after the indicated treatments. (B) Densitometric analysis of MuRF1 and MAFbx expression in cardiomyocytes. $\mathrm{n}=3$ for each group. ${ }^{*} \mathrm{P}<0.05$. ${ }^{~} \mathrm{P}<0.05$. MuRF1, muscle RING-finger protein-1; MAFbx, muscle atrophy F-box; Ang, angiotensin; Met, metformin.

and MAFbx-siRNA resulted in an average, 82 and $73 \%$ knockdown efficiency compared with control siRNAs, respectively (Figs. 4A, 4D and 4E). The results revealed that compared with those transfected with control siRNA, knocking down MuRF1 and MAFbx expression using siRNA significantly increased the cell surface area of cardiomyocytes in the presence of Ang II and metformin ( $\mathrm{P}<0.05$;Figs. 2 and 4C). Furthermore, the inhibitory effects of metformin on the Ang II-induced elevations of ANP and BNP mRNA and protein expression were significantly reversed by MuRF1-siRNA and MAFbx-siRNA transfection $(\mathrm{P}<0.05$; Fig. 4B, F and $\mathrm{G})$. Specifically, following Ang II and metformin treatment, the expression of ANP and BNP was also significantly higher in the MuRF1-siRNA and MAFbx-siRNA groups compared with that in the NC-siRNA group ( $\mathrm{P}<0.05$; Fig. 4B, F and $\mathrm{G})$. These results suggested that metformin upregulated the MuRF1 and MAFbx pathway to inhibit Ang II-induced cardiomyocyte hypertrophy (Figs. 2 and 4). 
A
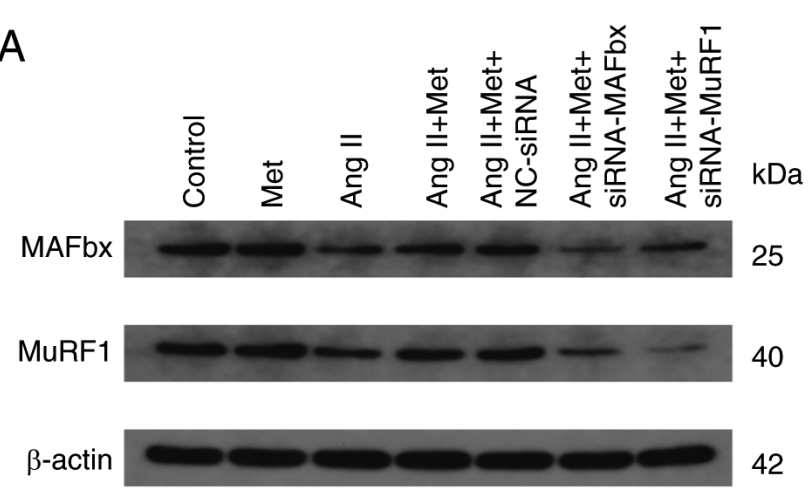

C
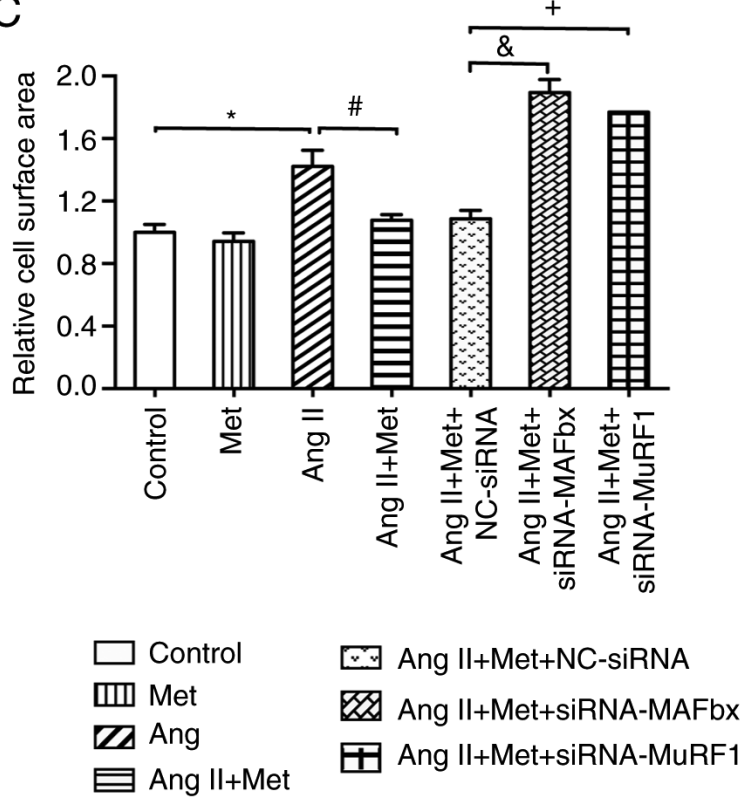

$\mathrm{F}$

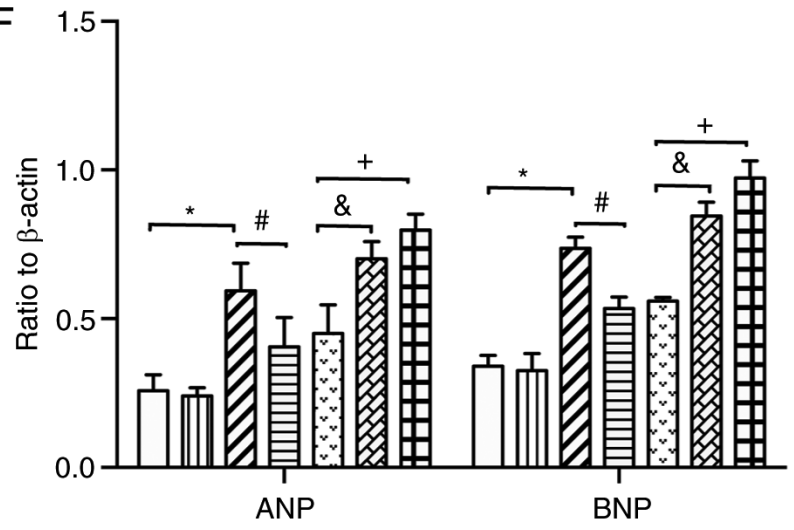

$\mathrm{B}$

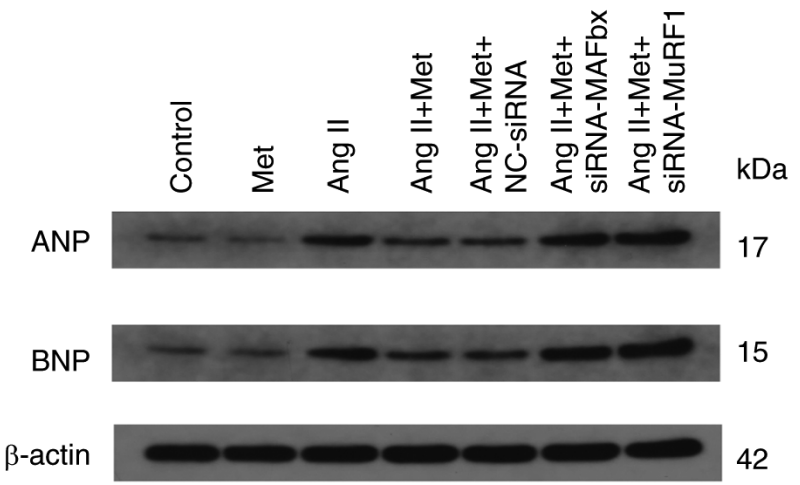

D

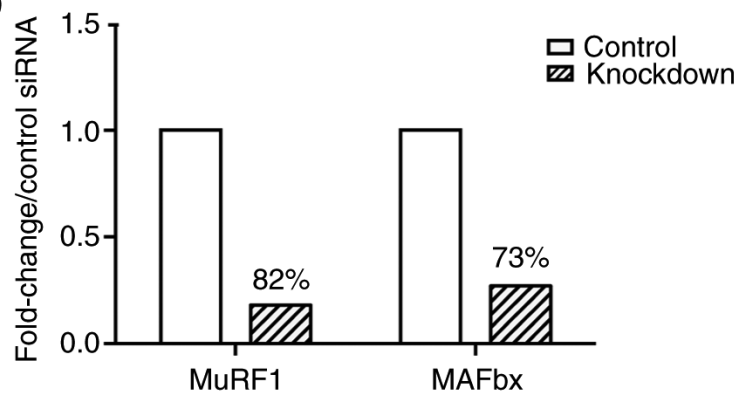

$E$

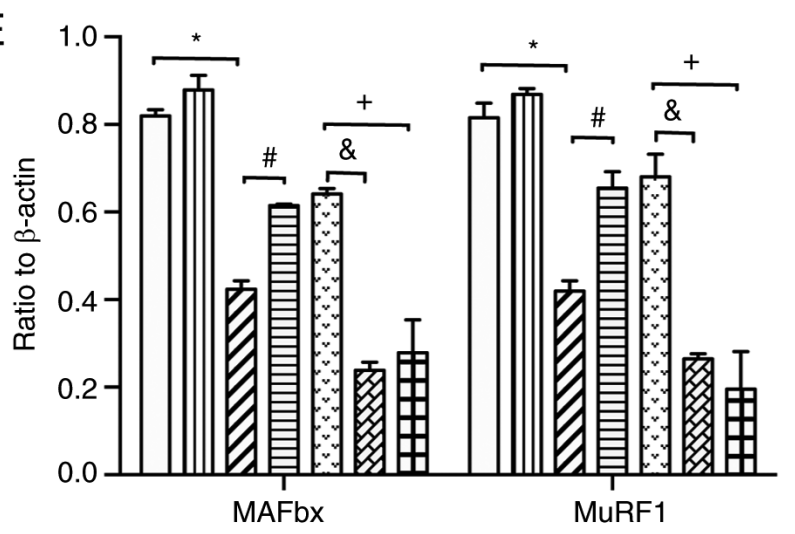

G

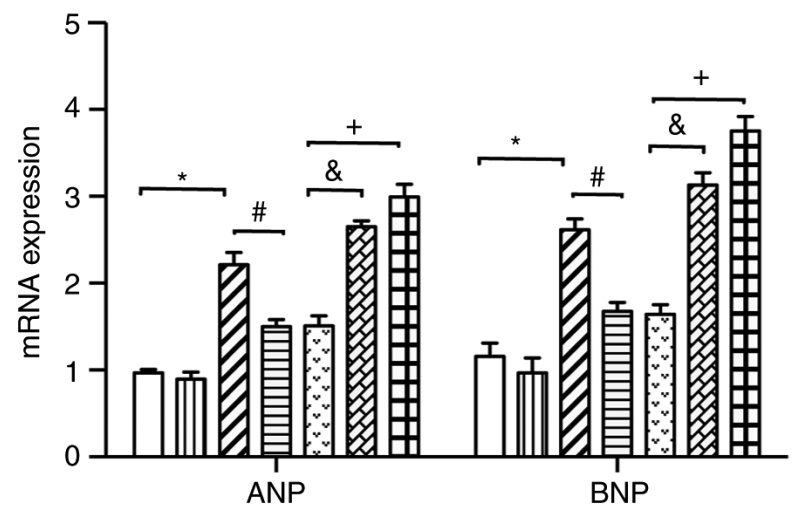

Figure 4. Effects of the MuRF1 and MAFbx signaling pathway on the effects of Metformin on Ang II-induced cardiomyocyte hypertrophy. (A-G) After treatment with Ang II and metformin, H9c2 cells were transfected with either NC-siRNA, MuRF1-siRNA or MAFbx-siRNA. In the presence of Ang II and metformin, MuRF1-siRNA and MAFbx-siRNA transfection (A) inhibit the expression of MuRF1 and MAFbx protein, (B) increase the expression of ANP and BNP protein and (C) increase cardiomyocyte size compared with NC-siRNA. (D) Measurement of MuRF1 and MAFbx expression following transfection with MuRF1-siRNA and MAFbx-siRNA by western blot presented in (A), which resulted in 82 and $73 \%$ knockdown efficiency compared with control siRNAs, respectively. (E) Densitometric analysis of MuRF1 and MAFbx expression presented in (A). (F) Densitometric analysis of ANP and BNP expression presented in (B). (G) MAFbx-siRNA and MuRF1-siRNA increases the mRNA expression of ANP and BNP. $\mathrm{n}=3$ for each group. ${ }^{*} \mathrm{P}<0.05$. ${ }^{\#} \mathrm{P}<0.05$. ${ }^{\mathrm{P}} \mathrm{P}<0.05$. ${ }^{+} \mathrm{P}<0.05$. Ang, angiotensin; MuRF1, muscle $\mathrm{RING}$-finger protein-1; MAFbx, muscle atrophy F-box; siRNA, small interfering RNA; ANP, atrial natriuretic peptide; BNP, brain natriuretic peptide; Met, metformin. 


\section{Discussion}

The present study investigated the potential effects of metformin on a model of cardiomyocyte hypertrophy and the underlying mechanism by which it functions. The results revealed that metformin attenuated cell hypertrophy by activating the MuRF1 and MAFbx pathway. It was also revealed that Ang II treatment significantly increased the surface area of cells and increased the mRNA and protein expression of ANP and BNP whilst reducing the mRNA and protein expression levels of MuRF1 and MAFbx. These data suggest that the MuRF1 and MAFbx signaling pathways may serve an important role in Ang II-induced cardiomyocyte hypertrophy.

MAFbx and MuRF1 are important factors in the UPS and have been studied widely in the context of cardiac hypertrophy $(9,10)$. MAFbx and MuRF1 are also considered to serve a role in protein degradation (17). Overexpression of MAFbx and MuRF1 may therefore lead to excessive protein degradation and muscle atrophy (18). Previous studies have suggested that MuRF1 and MAFbx were involved in the regulation of the hypertrophic response of cardiac tissue $(19,20)$. Reduced expression of MuRF1 and MAFbx in the myocardium have been documented to result in hypertrophy (21). Furthermore, previous studies have identified the pivotal role of certain E3 ubiquitin ligases in cardiac hypertrophy, including MuRF1 and MAFbx $(22,23)$. In particular, a number of studies have demonstrated that MuRF1 and MAFbx were activated during cardiac hypertrophy, where the precise control of myofiber synthesis and turnover was essential for maintaining structural integrity $(23,24)$.

Metformin is a first-line pharmacological agent used for the treatment of type 2 diabetes, which act to reduce the risk of cardiovascular events and even mortality (11). The beneficial effects of metformin on cardiac function have been attributed to direct actions on cell metabolism, endothelial function, platelet reactivity and calcium homeostasis (25). Since hypertrophic stimuli, including angiotensin II and pressure overload, may increase the expression of ANP and BNP (26), the mRNA levels of ANP and BNP following metformin and/or Ang II treatment were investigated as hypertrophic markers in the present study. The results revealed that metformin significantly suppressed the development of Ang II-induced cardiomyocyte hypertrophy as evidenced by reductions in cardiomyocyte surface area, reduced expression of hypertrophy-associated genes ANP and BNP in Ang II-stimulated cardiomyocytes. Metformin has also been demonstrated to serve cardioprotective effects by reducing left ventricular hypertrophy in patients with coronary artery disease, insulin resistance and pre-diabetes $(27,28)$ Additionally, previous studies have demonstrated that metformin can inhibit protein synthesis by activating MAPK, functioning in the anti-hypertrophic pathway in cardiomyocytes $(29,30)$.

The present study revealed that metformin increased the expression of MuRF1 and MAFbx. Furthermore, MuRF1 and MAFbx knockdown resulted in the reversal of metformin-mediated suppression of Ang II-induced cardiomyocyte hypertrophy. These results indicated that metformin inhibited cardiomyocyte hypertrophy by activating MuRF1and MAFbx signaling pathway. UPS-dependent protein degradation is an energy-consuming process, where
5'AMP-activated protein kinase (AMPK) serves a central role by regulating ATP production (31). Metformin has been previously demonstrated to increase AMPK activation to decrease Ang II-induced cardiac hypertrophy, whilst exerting cardioprotective effects (32). Consistent with this notion, the present results indicated that metformin conferred cardioprotective effects by regulating MuRF1and MAFbx expression. In addition, the present study demonstrated that MAFbx silencing was accompanied by markedly reduced MuRF1 expression, whereas MuRF1 silencing was also accompanied by markedly reduced MuRF1 expression. The reason for that remain unclear. Since both MAFbx and MuRF1 are crucial factors of $\mathrm{E} 3$ proteins that serve roles in protein degradation (22), the possible direct relationship between these two proteins require further study.

The expression of either MuRF1 or MAFbx is sufficient to inhibit cardiac hypertrophy (33). Previous studies have revealed that treatment with metformin increased MuRF1 and MAFbx transcription, in addition to increasing AMPK phosphorylation $(34,35)$. This suggest that AMPK activation results in the transcriptional activation of MuRF1 and MAFbx (36). In addition, previous studies have also demonstrated that AMPK activation by AMPK activator 5-aminoimidazole-4-carboxamide ribonucleoside (AICAR )significantly suppressed cardiomyocyte hypertrophy by increasing the activity of forkhead box protein $\mathrm{O} 1$ (FOXO1) and upregulating the expression of downstream atrogenes MuRF1 and MAFbx $(37,38)$. The effects of AICAR on cardiomyocyte hypertrophy were also inhibited after silencing MuRF1 and MAFbx $(37,38)$.

In conclusion, the present study revealed that the treatment of hypertrophied cardiomyocytes caused by Ang II with metformin attenuated cardiomyocyte hypertrophy through the MuRF1 and MAFbx pathway. The antihypertrophic effects of metformin may result from the upregulation of MuRF1 and MAFbx expression in cardiomyocytes. Although previous studies demonstrated that the AMPK/FOXO1 and MuRF1/MAFbx pathways may serve an important role in this process $(37,38)$, further studies are required for confirmation, especially in an in vivo setting. The AMPK/FOXO1 pathway was not measured in the present study, meaning that the mechanism by which AMPK/FOXO1 affects the metformin-induced attenuation of cardiomyocyte hypertrophy and MuRF1 and MAFbx transcriptional activity remain to be fully elucidated. Further studies are required to determine how the AMPK/FOXO1 and MuRF1/MAFbx pathways are regulated during cardiac hypertrophy.

\section{Acknowledgements}

Not applicable.

\section{Funding}

The present study was supported by the Guizhou Provincial Science and Technology Department Fund of China [grant no. Qian (2017)1103], The Science and Technology Fund of Guizhou Provincial Health Commission (grant nos. gzwjkj2018-1-005 and gzwjkj2019-1-097) and The Clinical Research Center Project of Department of Science \& Technology of Guizhou Province [grant no. (2017) 5405]. 


\section{Availability of data and materials}

The datasets used and/or analyzed during the current study are available from the corresponding author on reasonable request.

\section{Authors' contributions}

FD conducted the experiments, data collection and interpretation. BC participated in study design, coordination of the experiments and data collection. YR and YC analyzed and interpreted the data, QW was a major contributor in the conception and design of this study. All authors read and approved the final manuscript.

\section{Ethics approval and consent to participate}

Not applicable.

\section{Patient consent for publication}

Not applicable.

\section{Competing interests}

The authors declare that they have no competing interests.

\section{References}

1. Frey N and Olson EN: Cardiac hypertrophy: The good, the bad, and the ugly. Annu Rev Physiol 65: 45-79, 2003.

2. Horton JS, Shiraishi T, Alfulaij N, Small-Howard AL, Turner HC, Kurokawa T, Mori Y and Stokes AJ: 'TRPV1 is a component of the atrial natriuretic signaling complex, and using orally delivered antagonists, presents a valid therapeutic target in the longitudinal reversal and treatment of cardiac hypertrophy and heart failure'. Channels (Austin) 13: 1-16, 2019.

3. Patten RD and Hall-Porter MR: Small animal models of heart failure: Development of novel therapies, past and present. Circ Heart Fail 2: 138-144, 2009.

4. Huang L, Xi Z, Wang C, Zhang Y, Yang Z, Zhang S, Chen Y and Zuo Z: Phenanthrene exposure induces cardiac hypertrophy via reducing miR-133a expression by DNA methylation. Sci Rep 6: 20105, 2016.

5. Spänig S, Kellermann K, Dieterlen MT, Noack T, Lehmann S, Borger MA, Garbade J, Barac YD and Emrich F: The ubiquitin proteasome system in ischemic and dilated cardiomyopathy. Int J Mol Sci 20: 6354, 2019.

6. Lino CA, Demasi M and Barreto-Chaves ML: Ubiquitin proteasome system (UPS) activation in the cardiac hypertrophy of hyperthyroidism. Mol Cell Endocrinol 493: 110451, 2019.

7. Cacciapuoti F: Role of ubiquitin-proteasome system (UPS) in left ventricular hypertrophy (LVH). Am J Cardiovasc Dis 4: 1-5, 2014.

8. Cui X, Zhang Y, Wang Z, Yu J, Kong Z and Ružić L: High-intensity interval training changes the expression of muscle RING-finger protein-1 and muscle atrophy F-box proteins and proteins involved in the mechanistic target of rapamycin pathway and autophagy in rat skeletal muscle. Exp Physiol 104: 1505-1517, 2019.

9. Usui S, Chikata A, Takatori O, Takashima SI, Inoue O, Kato T, Murai H, Furusho H, Nomura A, Zablocki D, et al: Endogenous muscle atrophy F-box is involved in the development of cardiac rupture after myocardial infarction. J Mol Cell Cardiol 126: 1-12, 2019.

10. Gupta I, Varshney NK and Khan S: Emergence of members of TRAF and DUB of ubiquitin proteasome system in the regulation of hypertrophic cardiomyopathy. Front Genet 9: 336, 2018.

11. Marshall SM: 60 years of metformin use: A glance at the past and a look to the future. Diabetologia 60: 1561-1565, 2017.
12. Markowicz-Piasecka M, Huttunen KM, Mateusiak L, Mikiciuk-Olasik E and Sikora J: Is Metformin a Perfect Drug? Updates in Pharmacokinetics and Pharmacodynamics. Curr Pharm Des 23: 2532-2550, 2017.

13. Driver C, Bamitale KDS, Kazi A, Olla M, Nyane NA and Owira PMO: Cardioprotective Effects of Metformin. J Cardiovasc Pharmacol 72: 121-127, 2018.

14. Tseng YT: Cardioprotective effect of metformin against doxorubicin cardiotoxicity in rats. Anatol J Cardiol 16: 242-243, 2016.

15. Polianskyte-Prause Z, Tolvanen TA, Lindfors S, Dumont V, Van M, Wang H, Dash SN, Berg M, Naams JB, Hautala LC, et al: Metformin increases glucose uptake and acts renoprotectively by reducing SHIP2 activity. FASEB J 33: 2858-2869, 2019.

16. Tsai CH, Tsai HC, Huang HN, Hung CH, Hsu CJ, Fong YC, Hsu HC, Huang YL and Tang CH: Resistin promotes tumor metastasis by down-regulation of miR-519d through the AMPK/p38 signaling pathway in human chondrosarcoma cells. Oncotarget 6: 258-270, 2015.

17. Sun Y, Liang X, Chen J, Tang R, Li L and Li D: Change in Ubiquitin Proteasome System of Grass Carp Ctenopharyngodon idellus Reared in the Different Stocking Densities. Front Physiol 9: 837, 2018.

18. Menconi M, Gonnella P, Petkova V, Lecker S and Hasselgren PO: Dexamethasone and corticosterone induce similar, but not identical, muscle wasting responses in cultured L6 and $\mathrm{C} 2 \mathrm{C} 12$ myotubes. J Cell Biochem 105: 353-364, 2008.

19. Willis MS, Ike C, Li L, Wang DZ, Glass DJ and Patterson C: Muscle ring finger 1, but not muscle ring finger 2, regulates cardiac hypertrophy in vivo. Circ Res 100: 456-459, 2007.

20. Arya R, Kedar V, Hwang JR, McDonough H, Li HH, Taylor J and Patterson C: Muscle ring finger protein-1 inhibits PKC $\{$ epsilon\} activation and prevents cardiomyocyte hypertrophy. J Cell Biol 167: 1147-1159, 2004

21. Conraads VM, Vrints CJ, Rodrigus IE, Hoymans VY, Van Craenenbroeck EM, Bosmans J, Claeys MJ, Van Herck P, Linke A, Schuler G, et al: Depressed expression of MuRF1 and MAFbx in areas remote of recent myocardial infarction: A mechanism contributing to myocardial remodeling? Basic Res Cardiol 105: 219-226, 2010

22. Bodine SC, Latres E, Baumhueter S, Lai VK, Nunez L, Clarke BA, Poueymirou WT, Panaro FJ, Na E, Dharmarajan K, et al: Identification of ubiquitin ligases required for skeletal muscle atrophy. Science 294: 1704-1708, 2001.

23. Fielitz J, Kim MS, Shelton JM, Latif S, Spencer JA, Glass DJ, Richardson JA, Bassel-Duby R and Olson EN: Myosin accumulation and striated muscle myopathy result from the loss of muscle RING finger 1 and 3. J Clin Invest 117: 2486-2495, 2007.

24. Shaalan WM, El-Hameid NAA, El-Serafy SS and Salem M: Expressions and characterization of MuRFs, Atrogin-1, F-box 25 genes in tilapia, Oreochromis niloticus, in response to starvation. Fish Physiol Biochem 45: 1321-1330, 2019.

25. Nesti L and Natali A: Metformin effects on the heart and the cardiovascular system: A review of experimental and clinical data. Nutr Metab Cardiovasc Dis 27: 657-669, 2017.

26. Ying $\mathrm{H}, \mathrm{Xu} \mathrm{MC}$, Tan JH, Shen JH, Wang $\mathrm{H}$ and Zhang DF: Pressure overload-induced cardiac hypertrophy response requires janus kinase 2-histone deacetylase 2 signaling. Int J Mol Sci 15: 20240-20253, 2014.

27. Li J, Minćzuk K, Massey JC, Howell NL, Roy RJ, Paul S, Patrie JT, Kramer CM, Epstein FH, Carey RM, et al: Metformin Improves Cardiac Metabolism and Function, and Prevents Left Ventricular Hypertrophy in Spontaneously Hypertensive Rats. J Am Heart Assoc 9: e015154, 2020.

28. Mohan M, Al-Talabany S, McKinnie A, Mordi IR, Singh JSS, Gandy SJ, Baig F, Hussain MS, Bhalraam U, Khan F, et al: A randomized controlled trial of metformin on left ventricular hypertrophy in patients with coronary artery disease without diabetes: The MET-REMODEL trial. Eur Heart J 40: 3409-3417, 2019.

29. Rena G, Hardie DG and Pearson ER: The mechanisms of action of metformin. Diabetologia 60: 1577-1585, 2017.

30. Yang F, Qin Y, Wang Y, Meng S, Xian H, Che H, Lv J, Li Y, Yu Y, Bai Y, et al: Metformin inhibits the NLRP3 inflammasome via AMPK/mTOR-dependent effects in diabetic cardiomyopathy. Int J Biol Sci 15: 1010-1019, 2019.

31. Xiao B, Sanders MJ, Carmena D, Bright NJ, Haire LF, Underwood E, Patel BR, Heath RB, Walker PA, Hallen S, et al: Structural basis of AMPK regulation by small molecule activators. Nat Commun 4: 3017, 2013. 
32. Benes J, Kazdova L, Drahota Z, Houstek J, Medrikova D, Kopecky J, Kovarova N, Vrbacky M, Sedmera D, Strnad H, et al: Effect of metformin therapy on cardiac function and survival in a volume-overload model of heart failure in rats. Clin Sci (Lond) 121: 29-41, 2011.

33. Witt CC, Witt SH, Lerche S, Labeit D, Back W and Labeit S: Cooperative control of striated muscle mass and metabolism by MuRF1 and MuRF2. EMBO J 27: 350-360, 2008.

34. Krawiec BJ, Nystrom GJ, Frost RA, Jefferson LS and Lang CH: AMP-activated protein kinase agonists increase mRNA content of the muscle-specific ubiquitin ligases MAFbx and MuRF1 in C2C12 cells. Am J Physiol Endocrinol Metab 292: E1555-E1567, 2007.

35. Thomson DM: The Role of AMPK in the Regulation of Skeletal Muscle Size, Hypertrophy, and Regeneration. Int J Mol Sci 19: $3125,2018$.

36. Vilchinskaya NA, Krivoi II and Shenkman BS: AMP-Activated Protein Kinase as a Key Trigger for the Disuse-Induced Skeletal Muscle Remodeling. Int J Mol Sci 19: 3558, 2018.
37. Chen B, Wu Q, Xiong Z, Ma Y, Yu S, Chen D, Huang S and Dong Y: Adenosine monophosphate-activated protein kinase attenuates cardiomyocyte hypertrophy through regulation of FOXO3a/MAFbx signaling pathway. Acta Biochim Biophys Sin (Shanghai) 48: 827-832, 2016.

38. Chen BL, Ma YD, Meng RS, Xiong ZJ, Wang HN, Zeng JY, Liu C and Dong YG: Activation of AMPK inhibits cardiomyocyte hypertrophy by modulating of the FOXO1/MuRF1 signaling pathway in vitro. Acta Pharmacol Sin 31: 798-804, 2010 .

This work is licensed under a Creative Commons Attribution-NonCommercial-NoDerivatives 4.0 International (CC BY-NC-ND 4.0) License. 